\title{
Some Derived Sequences of K-Jacobsthal and K- Jacobsthal Lucas
}

\section{T. Ragunathan, G. Srividhya}

\begin{abstract}
In this article we present incredible associated some derived sequences of $k$ - Jacobsthal and $k$-Jacobsthal Lucas numbers and we give Diophantine triples for $(a, b, c)$ with the property $D\left(j_{k_{2} n-1}^{2}\right)$.
\end{abstract}

Key words: derived $k$-Jacobsthal numbers, derived k-Jacobsthal Lucas numbers

\section{INTRODUCTION}

Fibonacci sequence is the very popular for its magnificent and remarkable properties. In recent times, some of the sequences were generalized for any positive real number $k$. The studies of $k$-Fibonacci sequence, $k$-Lucas sequence, $k$-Jacobhstal and $k$-Jacobhstal-Lucas sequences etc., can be found.

S. Uygun, and H.Eldogan, defined $k$-jacobsthal and $k$-jacobsthal lucas sequences

$$
\begin{aligned}
& \quad j_{k, n}=k j_{k, n-1}+2 j_{k, n-2}, \quad j_{k, 0}= \\
& 0, j_{k, 1}=1, n \geq 2 \\
& \quad \text { and } \\
& \hat{\mathrm{j}}_{k, n}=k \hat{\mathrm{\jmath}}_{k, n-1}+2 \hat{\mathrm{\jmath}}_{k, n-2}, \quad \hat{\mathrm{J}}_{k, 0}=2, \hat{\mathrm{J}}_{k, 0}=k \\
& n \geq 2
\end{aligned}
$$

Give some properties of the $k$-Jacobsthal and k-jacobsthal Lucas sequence. S.Vidhayalaxmi M.A.Gopalan and E.Premalatha provides observations on derived $k$-Fibonacci and derived $k$ - Fibonacci Lucas sequences. Now , we bring in the some derived sequences of $k$-Jacobsthal and $k$-Jacobsthal Lucas numbers. Also give Diophantine triple for $(a, b, c)$ with property $D\left(j_{k, n-1}^{2}\right)$ and $D\left(\hat{\jmath}_{k, n-1}^{2}\right)$.

\section{Section I}

\footnotetext{
Revised Manuscript Received on December 15, 2019

T. Ragunathan, Department of Mathematics, PGP College of Arts and Science, Namakkal. Tamilnadu, India.

E-mail : math_ragu@rediffmail.com

G. Srividhya, Department of Mathematics, Government Arts College, Trichy-22, Tamilnadu, India.

E-mail : vkm292011@hotmail.com
}

\section{Derived $\boldsymbol{k}$-Jacobsthal Sequence}

For any positive real number $k$, the derived $k$-Jacobsthal sequence $\left\{j_{k_{2} n}\right\}_{n=1}^{\infty}$ is defined as $j_{k_{2} 0}=0, j_{k, 1}=1$ and

$j_{k_{2} n+1}=k j_{k, n}-2 j_{k, n-1}$ for $n \geq 2$ Binet form of for $j_{k_{2} n}$ is

$$
j_{k_{2} n}=\frac{\alpha^{n}-\beta^{n}}{\alpha-\beta} \text { where } \alpha+\beta=k_{2} \alpha \beta=-2
$$

\section{Derived $\boldsymbol{k}$-Jacobsthal Lucas Sequence}

The derived $k$-Jacobsthal Lucas sequence $\left\{\hat{\mathrm{J}}_{k_{2}}\right\}_{n=1}^{\infty}$ is defined as $\hat{\mathrm{j}}_{k, 0}=2, \hat{\mathrm{j}}_{k, 1}=k$ and

$\hat{\mathrm{j}}_{k, n+1}=k \hat{\mathrm{j}}_{k, n}-2 \hat{\mathrm{j}}_{k, n-1}$ for $n \geq 2$ Binet form of for $\hat{\mathrm{j}}_{k_{2} n}$ is

$$
\hat{\mathrm{j}}_{k_{2} n}=\alpha^{n}+\beta^{\mathrm{n}} \quad \text { where } \alpha+\beta=k_{\nu} \alpha \beta=-2
$$

\section{Derived $k$-Jacobsthal facts are prearranged}

$$
\begin{aligned}
& \text { 1. } j_{k, 2}=k \\
& \text { 2. } j_{k, 3}=k^{2}-2 \\
& \text { 3. } j_{k, 4}=k^{3}-4 k \\
& \text { 4. } j_{k, 5}=k^{4}-6 k^{2}+4 \\
& \text { 5. } j_{k, 6}=k^{5}-8 k^{3}+12 k \\
& \text { 6. } j_{k, 7}=k^{6}-10 k^{4}+24 k^{2}-8 \\
& \text { 7. } j_{k, 8}=k^{7}-12 k^{5}+40 k^{3}-32 k \\
& \text { 8. } j_{k, 9}=k^{8}-14 k^{6}+60 k^{4}-80 k^{2}+8 \\
& \text { 9. } j_{k, 10}=k^{9}-16 k^{7}+84 k^{5}-160 k^{3}+72 k \\
& \text { 10. } j_{k, 11}=k^{10}+18 k^{8}+36 k^{6}-280 k^{4}+232 k^{2}-16 \\
& \text { 11. } j_{k, 12}=k^{11}-20 k^{9}+68 k^{7}-296 k^{5}+352 k^{3}-160 k \\
& \text { 12. } j_{k, 13}=k^{12}-22 k^{10}+104 k^{8}-101 k^{6}+912 k^{4}-624 k^{2}+32 \\
& \text { 13. } j_{k, 14}=k^{13}-24 k^{11}+149 k^{9}-237 k^{7}+1404 k^{5}-1328 k^{3}+152 k \\
& \text { 14. } j_{k, 15}=k^{14}-26 k^{12}+193 k^{10}-445 k^{10}+1606 k^{6}-3152 k^{4}+1400 k^{2}-64
\end{aligned}
$$




\section{Some Derived Sequences of K-Jacobsthal and K- Jacobsthal Lucas}

\section{Derived $\boldsymbol{k}$-Jacobsthal Lucas facts are prearranged}

$$
\begin{aligned}
& \text { 1. } \hat{j}_{k, 2}=k^{2}-4 \\
& \text { 2. } \hat{j}_{k, 3}=k^{3}-6 k \\
& \text { 3. } \hat{j}_{k, 4}=k^{4}-8 k^{2}+8 \\
& \text { 4. } \hat{j}_{k, 5}=k^{5}-10 k^{3}+20 k \\
& \text { 5. } \hat{k}_{k, 1}=k^{6}-12 k^{4}+36 k^{2}-16 \\
& \text { 6. } \hat{k}_{k, 7}=k^{7}-14 k^{5}+56 k^{3}-56 k \\
& \text { 7. } \hat{j}_{k, 1}=k^{8}-16 k^{6}+80 k^{4}-128 k^{2}+32 \\
& \text { 8. } \hat{j}_{k, 9}=k^{9}-18 k^{7}+108 k^{5}-240 k^{3}+144 k \\
& \text { 9. } \hat{j}_{k, 10}=k^{10}-20 k^{8}+140 k^{6}-400 k^{4}+400 k^{2}-64 \\
& \text { 10. } \hat{j}_{k, 11}=k^{11}-22 k^{9}+158 k^{7}-508 k^{5}+640 k^{3}-208 k \\
& \text { 11. } \hat{j}_{k, 12}=k^{12}-24 k^{10}+198 k^{8}-788 k^{6}+1440 k^{4}-1008 k^{2}+128 \\
& \text { 12. } \hat{j}_{k, 13}=k^{13}-26 k^{11}+242 k^{9}-1104 k^{7}+2456 k^{5}-2288 k^{3}+544 k \\
& \text { 13. } \hat{j}_{k, 14}=k^{14}-28 k^{12}+290 k^{10}-1500 k^{8}+4032 k^{6}-5168 k^{4}+2560 k^{2}-256 \\
& \text { 14. } \hat{j}_{k, 15}=k^{15}-30 k^{13}+342 k^{11}-1984 k^{9}+6240 k^{7}-10080 k^{5}+7136 k^{3}-1344 k
\end{aligned}
$$

Properties of Derived $\boldsymbol{k}$-Jacobsthal and $\boldsymbol{k}$-Jacobsthal Lucas sequences

1. $j_{k_{2} n+1}-j_{k_{2} n-1}=\hat{\mathrm{j}}_{k_{2} n}$

2. $j_{k_{2}, 2 n}=j_{k, n} \hat{\mathrm{k}}_{k_{2} n}$

3. $\hat{\mathrm{j}}_{k, 2 n}-2^{n+1}=\left(\hat{\mathrm{j}}_{k, n}\right)^{2}$

4. $\hat{\mathrm{j}}_{k, 3 n}+3 \hat{\mathrm{j}}_{k, n}=\left(\hat{\mathrm{j}}_{k, n}\right)^{3}$

5. $\hat{\mathrm{j}}_{k_{2}, 2 n}=\left(k^{2}-8\right) \hat{\mathrm{j}}_{k, n}^{2}+2^{n+1}$

6. $\hat{j}_{k, 2 n+1}=\left(k^{2}-8\right) j_{k, n} j_{k, n+1}+2^{n} k$

7. $j_{k, n+1} j_{k, n-1}-\hat{\mathrm{j}}_{k, 2 n}=2^{n-1} k$

8. $\hat{\mathrm{y}}_{k, n}^{2}-\left(k^{2}-8\right) j_{k, n}^{2}=4.2^{n}$

9. $\hat{\mathrm{j}}_{k_{2} n}^{4}-\left(k^{2}-8\right)^{2} j_{k_{2} n}^{4}=16.2^{n \hat{j}_{k, 2 n}}$

$$
\hat{\mathrm{j}}_{k, 2 n}=\left(k^{2}-8\right) j_{k, n+1} j_{k, n-1}+2^{n}\left(k^{2}-\right.
$$

10.

$$
\text { 4) }
$$

11. $j_{k, m} \hat{\mathrm{j}}_{k, n}+j_{k, n} \hat{\mathrm{j}}_{k, m}=2 j_{k, m+n}$

12. $j_{k_{2} m} \hat{\jmath}_{k_{2} n}-j_{k_{2} n} \hat{\jmath}_{k_{2} m}=2^{n+1} j_{k_{2} m+n}$

13. $j_{k_{2} m} \hat{\mathrm{j}}_{k, n}=j_{k_{2} m+n}+2^{n} j_{k, m-n}$

14. $\hat{\mathrm{j}}_{k, m+n} \hat{\mathrm{j}}_{m-n}=\hat{\mathrm{j}}_{k, 2 m}+2 \hat{\mathrm{j}}_{k, 2 n}$

15. $\hat{\mathrm{j}}_{k_{2} m+n} j_{m-n}=j_{k_{2} m+n}-2^{n} \hat{\mathrm{j}}_{k_{3} m-n}$

\section{Section II}

The three distinct integers $a, b, c$ such that "product of any two from the set added with $j_{k_{2} n-1}^{2}$ is a perfect square". Let $a=j_{k_{2} n+1}$ and $b=\hat{\mathrm{j}}_{k_{2} n}$

Let $c$ be any non - zero integer.

Consider

$$
a c+j_{k_{2} n-1}^{2}=\beta^{2}
$$

This becomes

$$
j_{k, n+1} c+j_{k, n-1}^{2}=\beta^{2}
$$

as well

$$
\mathrm{b} c+j_{k, n-1}^{2}=\gamma^{2}
$$

provides

$$
\hat{\mathrm{j}}_{k_{2} n} c+j_{k_{2} n-1}^{2}=\gamma^{2}
$$

Using some algebra,

$$
\hat{\mathrm{j}}_{k_{2} n} \beta^{2}-j_{k_{2} n+1} \gamma^{2}=j_{k_{2} n-1}^{2}\left(\hat{\mathrm{j}}_{k_{2} n}-j_{k_{2} n+1}\right)
$$

with the linear transformations

$$
\begin{aligned}
& \beta=X+j_{k_{\imath} n+1} T \\
& \gamma=X+\hat{\jmath}_{k_{2} n} T
\end{aligned}
$$

and $\mathrm{T}=1$ we have

$$
X=j_{k, n-1}+j_{k_{\imath} n+1} \text { and }
$$

$\beta=2 j_{k, n+1}+j_{k_{2} n-1}$

From (1)

$$
c=4\left(j_{k, n-1}+j_{k, n+1}\right)
$$

hence $(a, b, c)$ is the Diophatine triple with the property $D\left(j_{k, n-1}^{2}\right)$.

\begin{tabular}{|l|l|l|l|l|}
\hline$n$ & $k$ & $a$ & $b$ & $c$ \\
\hline 1 & 1 & 1 & 1 & 4 \\
\hline 2 & 1 & 3 & 5 & 16 \\
\hline 3 & 1 & 5 & 7 & 24 \\
\hline
\end{tabular}

\section{Remark :}

In the same way the identical mode to apply $D\left(\mathrm{j}_{k, n-1}^{2}\right)$ and to find the Diophantine triples.

Let $a=\hat{\mathrm{\jmath}}_{k, n+1}$ and $b=j_{k_{2} n}\left(k^{2}+8\right)$

c

$$
=4\left(\hat{\mathrm{j}}_{k, n-1}+\hat{\mathrm{j}}_{k, n+1}\right)
$$




\begin{tabular}{|l|l|l|l|l|}
\hline$n$ & $k$ & $a$ & $b$ & $c$ \\
\hline 1 & 1 & 5 & 9 & 28 \\
\hline 2 & 1 & 7 & 9 & 32 \\
\hline 3 & 1 & 17 & 27 & 88 \\
\hline
\end{tabular}

\section{CONCLUSION}

In this paper we found derived sequences of $k$ Jacobsthal and $k$ - Jacobsthal Lucas numbers and we give Diophantine triples for $(a, b, c)$ with the property $D\left(j_{k, n-1}^{2}\right)$. Some exciting outcomes among the solutions are obtained.

\section{REFERENCES}

1. oradam A.F, Jacobsthal representation numbers, The Fibonacci Quarterly,34(1)(1996),45-54.

2. Koshy.T, Fibonacci and lucas numbers with Applications, John Wiley and sons Inc., NY,(2001)

3. Uygun.S, and Eldogan.H, Some properties of the k-Jacobsthal Lucas sequence Gen.Math.Notes, vol.36,No.1, pp 34-37.

4. Srividhya $\mathrm{G}$ and Ragunathan $\mathrm{T}$, Sequences of Diophantine Triples for $K$-Jacobsthal and K-Jacobsthal Lucas, International Journal of Pure and Applied Mathematics Volume 117 No. 12 2017, 431-439.

5. S.vidhyalaxmi,M.A.Goplan.,E.Premalatha, Observations of Derived $k$ - Fibonacci and Derived $k$-Fibonacci Lucas sequences, International journal of innovative Research in Science, Engineering and Technolgy, vol 5. Issue 8(2016) 15771-15778. 